\title{
Aberration Measurement and Correction in Scanning Transmission Electron Microscopy using Machine Learning
}

\author{
Ryusuke Sagawa $^{1}$, Fuminori Uematsu ${ }^{1}$, Keito Aibara ${ }^{1}$, Tomohiro Nakamichi ${ }^{1}$ and Shigeyuki Morishita ${ }^{2}$ \\ ${ }^{1}$ JEOL Ltd., Tokyo, Japan, ${ }^{2}$ JEOL Ltd., Akishima, Tokyo, Japan
}

Owing to the development of aberration correctors in electron microscopy, spatial resolution has been improved down to $\sim 40 \mathrm{pm}$ in scanning transmission electron microscopy (STEM) [1]. To achieve such high resolution, one has to perform a precise axis alignment of a microscope. There are two main steps in the alignment in STEM. The first one is a Ronchigram alignment which is performed by looking at the shape of it. In this alignment, not only higher order aberrations are corrected but also lower order aberrations such as defocus, two-fold astigmatism and axial coma aberration (P3) are roughly corrected. After this, a condenser lens aperture is inserted and then the lower order aberrations are finely corrected to finally obtain a nice STEM image. Although these steps are essential, users who are not familiar with Ronchigrams often find it difficult to perform the first alignment. For such users, we developed and have offered a software called JEOL $\mathrm{COSMO}^{\mathrm{TM}}$ in which recorded Ronchigrams were divided into sections and their auto-correlation functions were calculated, and finally residual aberrations are deduced and corrected from the two-dimensional shapes of the functions [2]. This software only requires Ronchigrams obtained from an amorphous area on any specimen and the calculation converges quickly. In this paper we aimed to speed up and enhance the accuracy of aberration measurement in JEOL COSMO ${ }^{\mathrm{TM}}$ more by integrating a machine learning regression model to it.

In machine learning, it is important to have enormous number of training data for a better estimation accuracy. For this reason, we have developed a simulator which can output Ronchigrams that reproduces the features of experimentally obtained ones very well (Figure 1). In the simulator, the effects of chromatic aberration, shot noise, and detector noise can be taken into account. As a proof-of-principle experiment, we first created simulated images for training data in which a variety of P3 were present and then created a regression model to estimate the values of the aberration using a convolutional neural network in the model structure. Although the absolute amplitude and the azimuthal angle of the aberration in the training data ranged from 0 to 6,000 $\mathrm{nm}$ and from 0 to $2 \pi$ radians, respectively, the estimated values from experimentally obtained Ronchigrams showed the precision of around $20 \%$ for both parameters. Because the technique only requires a single image of Ronchigram for each measurement, the precision is sufficiently high where a quick repetitive correction is possible. The developed model was then implemented in a real microscope system to test if it works properly. The microscope used was the NEOARM electron microscope (JEOL Ltd.) equipped with the ASCOR illumination corrector (CEOS GmbH) operated at $200 \mathrm{kV}$. The specimen was a thin amorphous Germanium film. The values of aberration estimated from a recorded Ronchigram were feedback to the corrector in real time to correct the aberration. Even though the initial P3 was very large as more than 3,000 nm of amplitude, the system finally corrected it down to less than $50 \mathrm{~nm}$ with the repetitions of around 4 to 5 times and within 10 seconds in most cases, which is better than a manual adjustment in terms of both speed and accuracy.

For a better estimate precision, it is important to accurately determine the values of higher order aberrations such as six-fold astigmatism (A6) and sixth-order three-lobe aberration (R7) as they affect the outer shape of Ronchigrams which is considered critical for determining the lower order aberrations such as P3, especially when they are small. To this end, we created another regression model to accurately estimate A6 and R7. Figure 2 shows that the Ronchigrams with estimated A6 and R7 (c) best resembled the ones that were experimentally obtained (b). Using the estimated values of A6 and R7, the precision of P3 estimation improved from $24 \%$ to $16 \%$. 
Our latest model can estimate P3 aberration even if there is another residual aberration such as two-fold astigmatism in Ronchigrams. In addition, they do not have to be recorded from a pure amorphous specimen but there can be some other materials (e.g. gold nanoparticles) on an amorphous film, which makes the model more robust for any type of specimen users might want to observe.
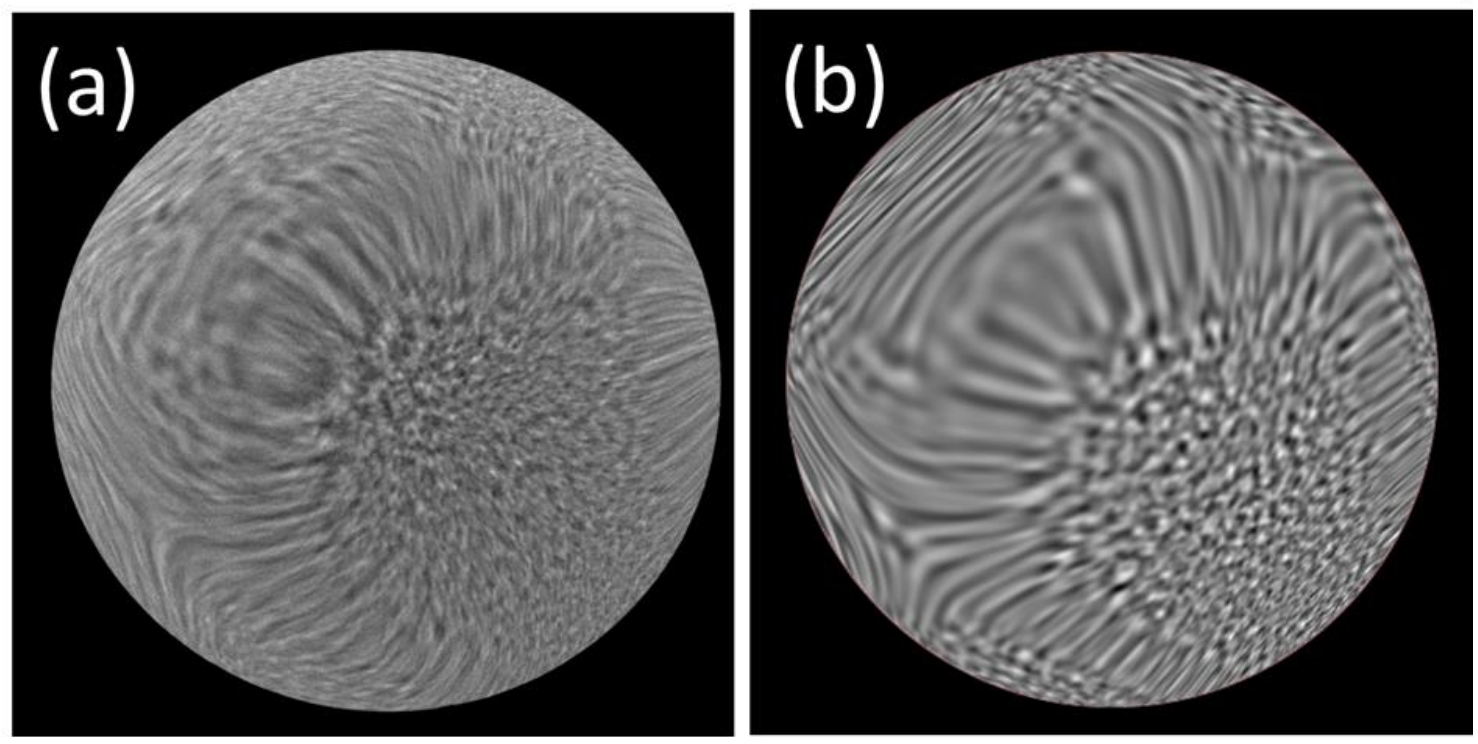

Figure 1. Figure 1. Comparison of Ronchigrams between (a) experimentally obtained and (b) simulated one.

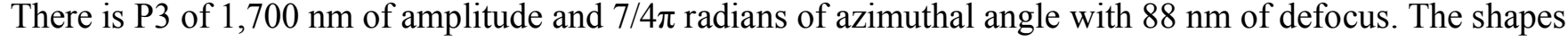
and the positions of stripes originated from an amorphous specimen are well reproduced in the simulated Ronchigram.
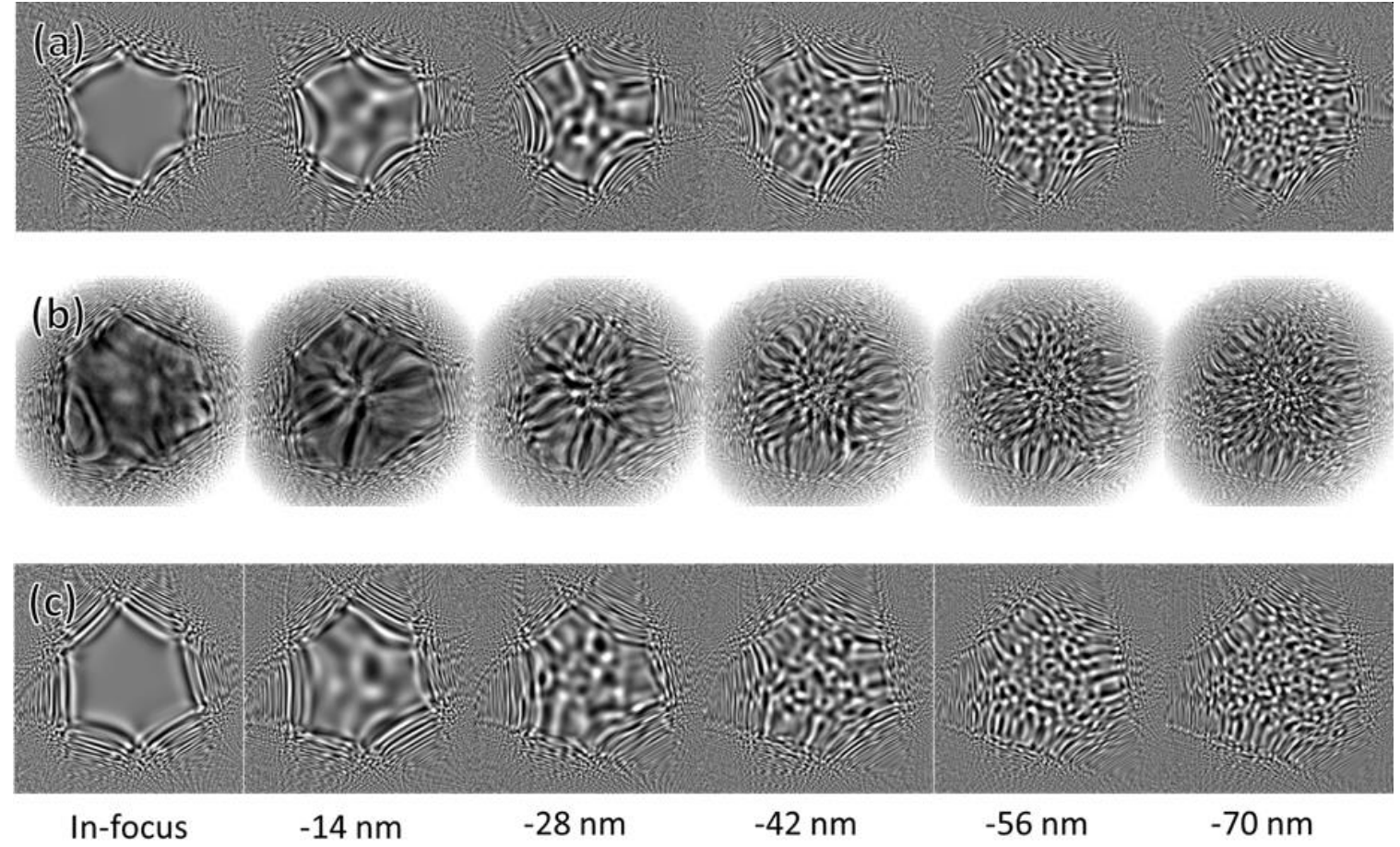

Figure 2. Figure 2. Comparison of Ronchigrams of (a) simulated images where A6 and R7 were manually (visually) determined, (b) experimentally obtained images and (c) simulated images where A6 and R7 were 
estimated by the regression model. The values at the bottom represent defocus. The amplitude and azimuthal angle of A6 and R7 were $0.1 \mathrm{~mm}, 40$ degrees and $5.0 \mathrm{~mm}, 5$ degrees, respectively in (a), and $0.038 \mathrm{~mm}, 22$ degrees and $3.3 \mathrm{~mm}, 7.2$ degrees, respectively in (c).

\section{References}

[1] S Morishita et al, Microscopy, 67 (2018) p. 46.

[2] H Sawada et al, Ultramicroscopy, 108 (2008) p. 1467. 